CERN-TH/97-184

IC $/ 97 / 93$

CPTH-S552.0797

hep-th/9708075

\title{
Duality in Superstring Compactifications with Magnetic Field Backgrounds ${ }^{\star}$
}

\author{
I. Antoniadis ${ }^{a, b}$, E. Gava ${ }^{c, d, e}$, K.S. Narain ${ }^{e}$ and T.R. Taylor ${ }^{f}$ \\ ${ }^{a}$ Theory Division, CERN, 1211 Geneva 23, Switzerland \\ ${ }^{b}$ Centre de Physique Théorique, Ecole Polytechnique, ${ }^{\dagger}$ F-91128 Palaiseau, France \\ ${ }^{c}$ Istituto Nazionale di Fisica Nucleare, sez. di Trieste, Italy \\ ${ }^{d}$ Scuola Internazionale Superiore di Studi Avanzati, Trieste, Italy \\ ${ }^{e}$ International Centre for Theoretical Physics, I-34100 Trieste, Italy \\ ${ }^{f}$ Department of Physics, Northeastern University, Boston, MA 02115, U.S.A.
}

\begin{abstract}
Motivated by the work of Polchinski and Strominger on type IIA theory, where the effect of non-trivial field strengths for $p$-form potentials on a Calabi-Yau space was discussed, we study four-dimensional heterotic string theory in the presence of a magnetic field on a 2-cycle in the internal manifold, for both $N=4$ and $N=2$ cases. We show that at special points in the moduli space, certain perturbative charged states become tachyonic and stabilize the vacuum by acquiring vacuum expectation values, thereby restoring supersymmetry. We discuss both the cases where the tachyons appear with a tower of Landau levels, which become light in the limit of large volume of the 2-cycle, and the case where such Landau levels are not present. In the latter case it is sufficient to restrict the analysis to the quartic potential for the tachyon. On the other hand, in the former case it is necessary to include the Landau levels in the analysis of the potential; for toroidal and orbifold examples, we give an explicit CFT description of the new supersymmetric vacuum. The resulting new vacuum turns out to be in the same class as the original supersymmetric one. Finally, using duality, we discuss the role of the Landau levels on the type IIA side.
\end{abstract}

${ }^{\star}$ Research supported in part by the National Science Foundation under grant PHY-96-02074, and in part by EEC under the TMR contracts ERBFMRX-CT96-0090 and FMRX-CT96-0012. ${ }^{\dagger}$ Laboratoire Propre du CNRS UPR A.0014.

CERN-TH/97-184

August 1997 


\section{Introduction}

In Ref. [四], breaking of $N=2$ supersymmetry in four dimensions was considered in type IIA theory as a consequence of non-trivial expectation values for various field strengths on the Calabi-Yau space. By choosing 2-, 4-, 6- and 10-form field strengths one could gauge the translational symmetry of the Neveu-Schwarz (NS) axion in all possible electric and magnetic directions. This leads to a scalar potential that comes from the gauge kinetic term of the ten-dimensional theory. With the exception of the 10-form field strength, for which one has to start from the massive type IIA theory, the remaining cases, for small field strengths, can be studied within the effective field theory starting from the conventional type IIA in $D=10$ and doing a Kaluza-Klein reduction. We recall that in the sigma-model frame the type IIA bosonic action in $D=10$ is:

$$
\begin{aligned}
S=\frac{1}{2} \int d^{10} x & {\left[\sqrt { - g } \left\{e^{-2 \varphi}\left[-R+4(\partial \varphi)^{2}-\frac{3}{4}(d B)^{2}\right]\right.\right.} \\
+ & \left.\left.\frac{1}{4} F_{2}^{2}+\frac{3}{4}\left(F_{4}-2 d B \wedge A\right)^{2}\right\}+\frac{1}{64} F_{4} \wedge F_{4} \wedge B\right],
\end{aligned}
$$

where $B$ is the NS-NS antisymmetric tensor field, $\varphi$ is the dilaton, and $F_{2}$ and $F_{4}$ are the field strengths of the Ramond-Ramond (R-R) 1-form and 3-form potentials, respectively.

Consider for instance, the case where the 6-form field strength $F_{6}$ (the dual of $F_{4}$ ) gets an expectation value $F_{6}=\nu_{6} \omega_{6}$, where $\omega_{6}$ is the volume form of the Calabi-Yau space and $\nu_{6}$ is a constant that is quantized in units of its inverse volume $V$. The ten-dimensional term $F_{6} \wedge F_{2} \wedge B$ will then give the four-dimensional coupling $A_{\mu} \partial_{\mu} a, A_{\mu}$ being the graviphoton gauge potential and $a$ the axion field. Thus, an expectation value of $F_{6}$ leads to an electric gauging of the translation symmetry of the axion. The resulting scalar potential, due to the kinetic energy of $F_{4}$, is $g_{I I}^{4} \nu_{6}^{2} V \sim g_{I I}^{4} / V$ (in the Einstein frame), where $g_{I I}=e^{\varphi}$ is the four-dimensional type IIA coupling. The fact that the coupling appears to the fourth power is due to the R-R nature of the gauge fields. This potential is runaway and vanishes only in the limit of infinite volume or vanishing coupling. However, it was argued that 
it can be stabilized in the presence of additional massless charged states in the unbroken theory [1]. In the type II theory, such massless states do indeed appear at the conifold points. In the presence of non-trivial field strengths these states become tachyonic and, after minimizing the quartic potential with respect to these tachyonic fields, one finds a new $N=2$ supersymmetric vacuum.

Our aim in this paper is to study the above phenomenon in the context of heterotic theory compactified on $K_{3} \times T^{2}$, where these tachyonic states appear at the perturbative level. As a result, one can carry out the analysis in a more precise and complete way. We will see below that in the presence of a magnetic field on a two-sphere in $K_{3}$, the tachyon state is in general accompanied by an infinite tower of excitations that correspond to higher Landau levels whose mass squares are of the order of the inverse power of the volume of the two-sphere. In the context of $N=2$ theories these higher Landau levels are non-BPS states and are therefore not stable. Nevertheless they contribute to the effective potential with terms of higher powers of the tachyon field that cannot be ignored in the large volume limit of the two-sphere, since the contributions they give to the vacuum energy are of the same order as the ones due to the quartic term. The large volume limit is mapped, via duality, to the weak coupling limit of IIA theory, where these higher Landau levels appear as branes on non-supersymmetric cycles whose masses are of the order of the coupling constant. The minimization of the potential therefore requires an analysis including all of these states.

We find that supersymmetry is restored at the new minimum and show that it is in the same class as the original supersymmetric vacuum before turning on the magnetic field. We will also study the case where all the excitations have mass splittings of the order of the string scale. In this case it is sufficient to restrict the analysis to the quartic terms in the tachyon effective potential, as higher powers in the tachyon field give, in the large volume limit, higher-order contributions to the vacuum energy.

The paper is organized as follows. In Section 2, guided by the type II-heterotic string 
duality, we show that the heterotic version of the phenomenon discussed in [1] involves turning on a magnetic field on appropriate two-cycles of the $N=2$ compactification manifold, $K_{3} \times T^{2}$, or $T^{6}$ in the $N=4$ case. For simplicity, we restrict ourselves to toroidal or orbifold compactifications. We also discuss the spectrum of states in the presence of a magnetic field. The tachyons that appear in the untwisted sector are accompanied by higher Landau levels, while those appearing in the twisted sector are not. In Section 3, we discuss the examples involving higher Landau levels both in $N=4$ and in $N=2$ theories. In the latter case, we discuss in some detail examples of $\mathbb{Z}_{2}$ and $\mathbb{Z}_{4}$ orbifold models. In Section 4, we study the case of twisted sector tachyons that do not appear with Landau levels. Section 5 contains a discussion of the implication of these results for the type IIA side.

\section{Heterotic Theory in the Presence of Magnetic Fields}

To anticipate what may happen in the heterotic string theory it is convenient to start from the six-dimensional (6D) type II-heterotic dual pair [2, 3]. Type IIA is compactified on $K_{3}$ while heterotic on $T^{4}$. The $6 \mathrm{D}$ gauge fields in type IIA are associated with the cohomology classes of $K_{3}$, namely one each from the 0 - and 4-cohomology and 22 from the 2-cohomology. On the heterotic side these gauge fields appear in the usual way: 4 from the left-moving fermionic sector and 20 from the right-moving bosonic sector. At a generic point in the moduli space, the gauge group is Abelian. Now let us further compactify the 6D theory on a two-dimensional compact space, which can be either $T^{2}$ or $C P^{1}$. In the $T^{2}$ case the resulting $4 \mathrm{D}$ theory has $N=4$ supersymmetry, while in the $C P^{1}$ case, in order to have conformal invariance, one must consider a fibration of the $6 \mathrm{D}$ theory over the base $C P^{1}$ and the resulting $4 \mathrm{D}$ theory has $N=2$ supersymmetry $[4,6$, 6]. In the type IIA case this gives rise to a Calabi-Yau space, which is a $K_{3}$ fibration over the base, while in the heterotic case, this can be thought of as a compactification on $K_{3} \times T^{2}$, together with an 
appropriate gauge bundle. The base $C P^{1}$ in this case is part of $K_{3}$. Under the duality, the $4 \mathrm{D}$ inverse coupling squares of type II $\left(g_{I I}^{-2}\right)$ and heterotic $\left(g_{H}^{-2}\right)$ are mapped to the volume of the base of the heterotic $\left(V_{H}\right)$ and type II $\left(V_{I I}\right)$, respectively.

Thus, if one wants to describe the above phenomenon of supersymmetry restoration perturbatively on the heterotic side, one should consider those gaugings on the type IIA side for which the scalar potential vanishes in the limit of large volume of the base. If we give expectation values to the field strengths of the $6 \mathrm{D}$ gauge potentials (that survive the fibration) on the base, then these field strengths are quantized via the Dirac quantization condition. In the limit of large $V_{I I}$, where the metric of the Calabi-Yau factorizes into a component along the fibre and one along the base, one can see that the field strength is quantized in units of the inverse volume $V_{I I}$ of the base. The kinetic term for the gauge field then gives rise to a $4 \mathrm{D}$ tree-level potential. As we mentioned above, in the large $V_{I I}$ limit, the resulting potential (in the Einstein frame) is of order $g_{I I}^{4} / V_{I I}$. From the $D=10$ point of view, these gauge field strengths correspond to 2-, 4- and 6-forms that have nonvanishing integrals over the base. On the heterotic side, such field strengths will have the interpretation of 6D gauge fields, acquiring non-vanishing field strengths on the base.

On the heterotic side there is a $6 \mathrm{D}$ tree-level coupling $d B \cdot \omega(A)$, with $B$ being the antisymmetric tensor and $\omega$ the Chern-Simons form. It follows that a non-trivial field strength on the base induces in four dimensions a term $A_{\mu} \partial_{\mu} b$, where $b$ is the pseudoscalar modulus associated to the Kähler class of the base. This mechanism therefore gauges the translation symmetry of $b$. This is expected from the fact that the type IIA axion is mapped to $b$ under duality. I Note that in the heterotic side this gauging is always electric.

By the usual Dirac quantization condition, the expectation value of the field strength is quantized in units of the inverse volume $V_{H}$ of the base, in the limit of large $V_{H}$. Note

\footnotetext{
${ }^{1}$ This translation symmetry is of course broken by world-sheet instanton effects on the heterotic side, in the same way as the axion shift is broken by space-time non-perturbative effects on the type II side.
} 
that under duality the modulus $V_{H}$ is mapped to the type II dilaton and belongs to a hypermultiplet in the case of $N=2$ compactifications. The $6 \mathrm{D}$ gauge kinetic term then gives rise to a tree-level cosmological constant that falls off as $g_{H}^{2} / V_{H}^{2}$ (in the Einstein frame). Taking into account the relation between coupling and volume in the two theories, we see that their scalar potentials match one another. As discussed in Ref. [1], this runaway potential can be stabilized if there are special points in the vector moduli space at which there appear extra massless states, which are charged under the above gauge field. In heterotic perturbation theory, such states can arise only for gauge fields coming from the right-moving sector. In the following we will restrict ourselves to such gaugings.

In Refs. [7, 8, 9], the spectrum in the presence of a constant magnetic field $F$ for some $U(1)$ gauge field on a torus of volume $V$ has been analysed. Given the minimum charge $q_{\text {min }}$, the single-valuedness of the wave function of charged states implies the following quantization for the magnetic field:

$$
F=\frac{2 \pi k}{q_{\min } V}
$$

for some integer $k$. Then, the previously massless states, with charge $q=\ell q_{\text {min }}$ in $D=6$, give rise to a tower of Landau levels with masses (for small $F$ ):

$$
M^{2}=g_{H}^{2}[(2 n+1)|q F|-2 s q F]
$$

and multiplicity $\ell k$. Here, $s$ is the eigenvalue of the internal spin operator, and the nonnegative integer $n$ labels the Landau levels. Landau levels arise from the quantization of the two compact momenta that do not commute in the presence of a magnetic field. In the toroidal (or orbifold) case there is always a state (untwisted) with $s=1$; for $n=0$, this state becomes tachyonic, with mass squared $-g_{H}^{2}|q F|$. Note that for fermions $s= \pm 1 / 2$, the two signs corresponding to the left and right chirality in $D=4$, respectively. Thus one chirality (say left) fermion (for $n=0$ ) remains massless while the other chirality (right) becomes massive and is paired with the left part of $n=1$ fermion and so on. These results, 
obtained from field theory considerations, can also be verified in the context of type I string theory, and the tree-level mass formula has been obtained to all orders in $F$ in Refs. [8, 10].

In the case of orbifold compactifications, there is also another class of massless charged states, hypermultiplets arising from the twisted sector, which have no six-dimensional interpretation. In the presence of a magnetic field, these states can also become tachyonic; however, they are not accompanied by Landau levels with mass splittings proportional to $F$ because they have no momenta in the $K_{3}$ direction. This is most easily seen by going to the type I side [11, 12, 13]. Here the gauge group arises from 9- and 5-brane Chan-Paton charges, with the 9-brane gauge group corresponding to the heterotic perturbative gauge group. The 5-branes are located at fixed points of the $K_{3}$ orbifold. The heterotic twisted orbifold states are mapped to ground states of open strings stretched between 9- and 5branes. Note that these strings have mixed Neumann-Dirichlet (ND) boundary conditions; therefore they do not carry Kaluza-Klein $K_{3}$ momentum, similarly to the heterotic case. The magnetic field is now on a 9-brane plane, orthogonal to the 5-branes. The effect of such a field on 9-brane is to modify the boundary condition of the open string on the 9-brane end from Neumann to a generalized boundary condition. Therefore the 95 string, that involved half-integer oscillator modes in the ND directions, now involves oscillators with frequencies $(1 / 2 \pm q F)$ modulo integers (in the weak field approximation). As a result, the zero point energy gets shifted and the previously massless scalars split into two states $\chi$, $\eta$, with masses:

$$
M_{\chi}^{2}=-|q F|, \quad M_{\eta}^{2}=|q F|,
$$

in the type I string frame, while the fermions remain massless. Since the lowest oscillator mode has a frequency $(1 / 2-|q F|)$, it is clear that the excited states have mass splittings of order the string scale and as a result one does not have the usual Landau levels.

As we will see below, since the presence of Landau levels affects the analysis of the potential, we will consider the cases with and without them separately. 


\section{Tachyons with Landau Levels}

This case includes $N=4$ toroidal compactification, as well as untwisted sector in $N=2$ orbifold compactifications of the heterotic string. Explicitly, in the untwisted sector, the tachyonic modes corresponding to $s= \pm 1$ in Eq. (2.2) are $\left[\left(\partial X_{4} \pm i \partial X_{5}\right) e^{i P_{L} \cdot X_{L}+i P_{R} \cdot X_{R}}+\right.$ the supersymmetric completion], where $X_{4}, X_{5}$ are the coordinates of the torus, and $P_{L}$ and $P_{R}$ are charges of the gauge fields coming from left- and right-moving sectors, respectively. These charges satisfy the condition $P_{R}^{2}-P_{L}^{2}=2$. The squared masses of these states are $\frac{1}{2} P_{L}^{2}$. In the presence of the constant gauge field strength $F_{45}^{a}$ in some right-moving direction labelled by $a$, these masses are shifted according to Eq. (2.2), with $q=P_{R}^{a} / \sqrt{2}$. By adjusting the Wilson lines, one can go to a point in the moduli space where $P_{L}=0$ and then the mass is just given by Eq. (2.2). In fact there are at least two states (or an even number) with the same mass, and they correspond to $P_{R} \rightarrow-P_{R}$ and $s \rightarrow-s$. As a result the massless fermions are paired and become non-chiral. This was to be expected, because by turning on the Wilson lines the fermion can pick up a mass. Note that in this way we can never get a situation like the conifold, where only one hypermultiplet becomes massless.

The existence of tachyons in the spectrum signals instability; dynamically the tachyon will start getting an expectation value. The question we would like to address is whether there exists a new critical point where the potential vanishes and the supersymmetry is restored. In order to get a feeling for what might happen, let us consider the effective potential of the tachyon up to the quartic term. To leading order in $1 / V$, this quartic potential arises from the Kaluza-Klein reduction of a 6D gauge theory, in the presence of a background magnetic field on the base. Since the tachyons in question involve internal spins $s= \pm 1$, the relevant gauge theory is that of $S U(2)$, where the internal components of $W^{ \pm}$with internal helicities $s= \pm 1$ play the role of the tachyon. In the following we first discuss the $N=4$ case, where the base manifold is $T^{2}$, and then discuss the modifications 
for the $N=2$ case, when the base is realized as an orbifold.

\section{1. $N=4$ Case}

The discussion in this case is very similar to the one appearing in the bound-state problem of $p$ - and $(p+2)$-branes [14]. Since modular invariance implies that the complete spectrum must also include fundamental representations of $S U(2)$, the minimum value of the background magnetic field is $\frac{2 \pi}{V} \sigma_{3}$, with $\sigma_{3}$ the Pauli matrix. This can be accomplished for instance by choosing the background gauge potential to be $A_{5}^{0}=\frac{2 \pi}{V} \frac{x_{4}}{R_{4}} \sigma_{3}$ and all the remaining $A$ 's equal to zero. Note that this background field satisfies the periodicity conditions:

$$
\begin{array}{ll}
A^{0}\left(x_{4}, x_{5}+R_{5}\right)=\Omega_{1} A^{0}\left(x_{4}, x_{5}\right), & \Omega_{1}=1 \\
A^{0}\left(x_{4}+R_{4}, x_{5}\right)=\Omega_{2} A^{0}\left(x_{4}, x_{5}\right), & \Omega_{2}=e^{2 i \pi x_{5} \sigma_{3} / R_{5}}
\end{array}
$$

where the symbol $\Omega A$ means the gauge transformation of $A$ by $\Omega$. Note that this boundary condition corresponds to a trivial $\mathbb{Z}_{2}$ flux:

$$
\Omega_{1}(0,0) \Omega_{2}\left(0, R_{5}\right)=\Omega_{2}(0,0) \Omega_{1}\left(R_{4}, 0\right)
$$

This fact will play an important role later.

Let us parametrize the fluctuations of $A_{4}$ and $A_{5}$ (i.e. the scalars in $4 \mathrm{D}$ space-time) around this background in the following way:

$$
A_{z}=A_{z}^{0}+\phi \sigma_{3}+\chi \sigma_{+}+\bar{\eta} \sigma_{-}, \quad A_{\bar{z}}=\left(A_{z}\right)^{\dagger}
$$

where $z=x_{5}+i x_{4}$ and $\sigma_{ \pm}=\sigma_{1} \pm i \sigma_{2}$. The boundary conditions on $\phi, \chi$ and $\eta$ are determined from the fact that $A$ in Eq. (3.3) satisfies the same boundary conditions as the background field, namely Eq. (3.1). This means that under $x_{5} \rightarrow x_{5}+R_{5}$ they remain unchanged while under $x_{4} \rightarrow x_{4}+R_{4}$ they transform as:

$$
\phi \rightarrow \phi, \quad \chi \rightarrow e^{4 \pi i x_{5} / R_{5}} \chi, \quad \eta \rightarrow e^{4 \pi i x_{5} / R_{5}} \eta
$$


To leading order in the large volume limit, the potential energy is given by $\int_{T^{2}} \operatorname{tr} F^{2}$, where $F$ is the field strength along the $T^{2}$ directions. Explicitly, the components of $F$ are:

$$
\begin{aligned}
& F_{3}=\frac{2 \pi}{V}-i\left(\partial_{\bar{z}} \phi-\partial_{z} \bar{\phi}\right)+|\eta|^{2}-|\chi|^{2} \\
& F_{+}=D_{z} \chi+D_{z}^{\dagger} \eta+2 i \bar{\phi} \chi-2 i \phi \eta
\end{aligned}
$$

where the subscripts in $F$ are the gauge indices, $D_{z}=\partial_{z}-2 i x_{4} / V$, and $D_{z}^{\dagger}=-\partial_{\bar{z}}+2 i x_{4} / V$ is the adjoint of $D_{z}$. From the analogy with the harmonic oscillator problem, it is clear that $D_{z}$ acts as an annihilation operator while its adjoint $D_{z}^{\dagger}$ acts as the creation operator. The ground state that is annihilated by $D_{z}$ and subject to the boundary condition (3.4) gives the two degenerate tachyon wave functions:

$$
\Psi_{0}^{(1)}=N e^{-\pi x_{4}^{2} / V} \theta_{2}(\tau \mid \bar{z}) \quad ; \quad \Psi_{0}^{(2)}=N e^{-\pi x_{4}^{2} / V} \theta_{3}(\tau \mid \bar{z}) \quad ; \quad \tau \equiv 2 i \frac{R_{4}}{R_{5}}
$$

where $\theta$ 's are the Jacobi theta-functions and $N=(-2 i \pi \tau)^{1 / 4}$ is a normalization factor such that $\int_{T^{2}}\left|\Psi_{0}^{(i)}\right|^{2}=V$ for $i=1,2$. Their mass is given by Eq. (2.2) for $n=0$ and $s=1$. The wave functions for higher Landau levels are simply obtained by applying the creation operators $D_{z}^{\dagger}$ on $\Psi_{0}^{(i)}$ and using the background gauge condition $D_{z} \chi=D_{z}^{\dagger} \eta$.

The $\chi$ field can then be expanded in terms of orthonormal wave functions $\Psi_{n}^{(i)}$ 's obtained by applying the creation operators $D_{z}^{\dagger^{n}}$ on $\Psi_{0}^{(i)}$. Thus, $\chi=\sum_{n} \chi_{n}^{(i)} \Psi_{n}^{(i)}$, with some complex coefficients $\chi_{n}^{(i)}$ that play the role of four-dimensional scalar fields. Similarly, one can expand the field $\eta=\sum_{n} \eta_{n}^{(i)} \Psi_{n}^{(i)}$. The background gauge condition can be used to solve $\eta$ in terms of $\chi$ with the result $\eta_{n}^{(i)}=\sqrt{\frac{n+2}{n+1}} \chi_{n+2}^{(i)}$, while one also finds that $\chi_{1}^{(i)}=0$. Substituting these expansions in the action and rescaling $\chi_{n+2}^{(i)} \rightarrow \sqrt{\frac{n+1}{2 n+3}} \chi_{n+2}^{(i)}$ in order to get standard kinetic terms, we find that their masses are $(2 n+3) g_{H}^{2} / V$, in agreement with the mass fomula (2.2). In fact, Eq. (2.2) with $s= \pm 1$ implies that these states come with multiplicity 4 while the tachyon and the first excitation with mass $g_{H}^{2} / V$ are twofold-degenerate. On the other hand, the transverse vectors corresponding to $s=0$ are twofold-degenerate and have masses $(2 n+1) g_{H}^{2} / V$. Combining all these states into massive Lorentz representations, one 
finds that there are two massive spin 1 representations for each level $(2 n+1) g_{H}^{2} / V$ and two scalars for each level $(2 n+3) g_{H}^{2} / V$, besides the tachyons. These scalars are precisely the ones we have found above by mode expansion and which enter in the minimization of the scalar potential.

The background gauge condition for the field $\phi$ is $\partial_{\bar{z}} \phi+\partial_{z} \bar{\phi}=0$. Since $\phi$ is periodic on the torus as seen from Eq. (3.4), its mode expansion subject to the gauge condition is given by:

$$
\phi=\phi_{0}+i \sum_{p \neq 0} \arg (p) \phi_{p} e^{2 i \pi p \cdot x} \quad ; \quad \phi_{-p}=\bar{\phi}_{p},
$$

with momenta $p \equiv\left(p_{4}, p_{5}\right)=\left(m_{4} / R_{4}, m_{5} / R_{5}\right)$ for integers $m_{4,5}$ and $\arg (p)=\arctan \left(p_{4} / p_{5}\right)$. The mass of the mode $\phi_{p}$ is $g_{H}^{2}|p|$.

The tachyon effective potential up to the quartic level has an irreducible part, which can be read off from $\int_{T^{2}} \operatorname{tr} F^{2}$ :

$$
\begin{aligned}
\mathcal{V}_{\mathrm{eff}}^{\mathrm{irr}}= & \frac{1}{2} g_{H}^{2}\left\{\left(\frac{2 \pi}{V}\right)^{2}-\frac{4 \pi}{V}\left(\left|\chi_{0}^{(1)}\right|^{2}+\left|\chi_{0}^{(2)}\right|^{2}\right)+\theta_{3}(\tau) \theta_{3}(-1 / \tau)\left(\left|\chi_{0}^{(1)}\right|^{4}+\left|\chi_{0}^{(2)}\right|^{4}\right)\right. \\
& \left.+4 \theta_{3}(\tau) \theta_{4}(-1 / \tau)\left|\chi_{0}^{(1)}\right|^{2}\left|\chi_{0}^{(2)}\right|^{2}+2 \theta_{2}(\tau) \theta_{4}(-1 / \tau)\left[\left(\chi_{0}^{(1)} \bar{\chi}_{0}^{(2)}\right)^{2}+\text { c.c. }\right]\right\}
\end{aligned}
$$

where the appearance of $\theta$-functions is a result of integration of the four-tachyon wave functions (3.6). In addition to these irreducible terms, the effective potential also receives reducible contributions due to the exchange of the Kaluza-Klein modes $\phi_{p}$ 's through the following 3-point vertex:

$$
C_{p}^{(i, j)} \phi_{p} \chi_{0}^{(i)} \bar{\chi}_{0}^{(j)} \quad, \quad C_{p}^{(i, j)}=|p| e^{-i \pi m_{4}\left(\frac{m_{5}}{2}-j\right)} e^{i \frac{\pi}{2}\left(\frac{p_{5}^{2}}{4} \tau-\frac{p_{4}^{2}}{\tau}\right)} \quad ; \quad m_{5}+i-j=\text { even },
$$

where the structure constants $C_{p}^{(i, j)}$ arise upon integration of the appropriate wave functions, and they vanish if $p_{5}$ does not satisfy the above condition. It is easy to check that the contribution of the reducible diagrams is:

$$
\begin{aligned}
\mathcal{V}_{\mathrm{eff}}^{\mathrm{red}}= & \frac{1}{2} g_{H}^{2}\left\{\left[1-\theta_{3}(\tau) \theta_{3}(-1 / \tau)\right]\left(\left|\chi_{0}^{(1)}\right|^{4}+\left|\chi_{0}^{(2)}\right|^{4}\right)+\left[2-4 \theta_{3}(\tau) \theta_{4}(-1 / \tau)\right]\left|\chi_{0}^{(1)}\right|^{2}\left|\chi_{0}^{(2)}\right|^{2}\right. \\
& \left.-2 \theta_{2}(\tau) \theta_{4}(-1 / \tau)\left[\left(\chi_{0}^{(1)} \bar{\chi}_{0}^{(2)}\right)^{2}+\text { c.c. }\right]\right\}
\end{aligned}
$$


As a result, the effective potential up to this order becomes a total square:

$$
\mathcal{V}_{\text {eff }}=\frac{1}{2} g_{H}^{2}\left(\frac{2 \pi}{V}-\left|\chi_{0}^{(1)}\right|^{2}-\left|\chi_{0}^{(2)}\right|^{2}\right)^{2}
$$

Now it is clear that the minimum of the potential is at $\sum_{i}\left|\chi_{0}^{(i)}\right|^{2}=2 \pi / V$ and that at this point the potential vanishes.

Let us now consider the higher-order terms in the tachyon effective potential. These terms can either come from higher-derivative terms in $D=6$ (e.g. $F^{4}$ terms) or from reducible diagrams of higher-point functions. It is easy to see that all the contributions arising from the higher-derivative interactions are suppressed in the large volume limit. However, the reducible diagrams involving vertices coming from $F^{2}$ lead to contributions that are of the same order in volume as the quartic potential considered above. Indeed, the $n$-point vertex scales as $V^{\frac{n-4}{2}}$, while the propagators of the massive fields scale as $V$. A simple counting then shows that $\left|\chi_{0}\right|^{2 n}$ scales as $V^{n-2}$. The fact that the reducible diagrams involving exchanges of massive fields $\phi_{p}$ 's and the higher Landau levels $\chi_{n}$ 's contribute just means that these fields also acquire expectation values. It is therefore more efficient to analyse the minimization problem directly at the six-dimensional level.

Since the potential $\int_{T^{2}}\left(F_{3}^{2}+\left|F_{+}\right|^{2}\right)$ is a sum of integrals of semi-positive definite functions, the only way the potential can vanish is if each function vanishes individually. This means that the field strengths F's must vanish. The question therefore is whether one can continuously change the $S U(2)$ field strength from the original non-zero value to a vanishing one respecting the boundary conditions (3.1) and (3.2). This has been shown to be possible in Refs. [15, 16] for general toron boundary conditions. In fact, our case (3.2) corresponds to the trivial $\mathbb{Z}_{2}$ flux. By a suitable $S U(2)$ gauge transformation one can set the boundary conditions $\Omega_{1}$ and $\Omega_{2}$ to be constant commuting group elements. The effect of these constant commuting elements consists in turning on appropriate Wilson lines. As a result the new vacuum belongs to the same class as the one of the original supersymmetric theory, and the effect of the magnetic field is completely washed out. 


\section{2. $N=2$ Case}

Although the above discussion was made for the case of toroidal compactification (i.e. $N=$ 4 theory), it can be extended to the case of the untwisted sector in orbifold compactifications that give $N=2$ theories, provided the tachyons are not projected out by the orbifold group. To be concrete, we consider here the examples of $\mathbb{Z}_{2}$ and $\mathbb{Z}_{4}$ orbifolds [17]. In the former case the would-be tachyons appear in pairs, which gives an extra flat direction in the supersymmetric theory, while in the latter case there is only one charged massless hypermultiplet at the special point in the moduli space, which provides a tachyonic mode in the presence of a magnetic field. As a result there is no flat direction associated to it.

\section{$\mathbb{Z}_{2}$ orbifold}

Consider the theory in $D=4$ obtained by compactifying the ten-dimensional theory on $\left(T^{2} \times T^{2} / \mathbb{Z}_{2}\right) \times T^{2}$ together with a shift $\delta$ in one of the $E_{8}$ 's subject to the condition $2 \delta \in \Gamma_{8}$, where $\Gamma_{8}$ is the $E_{8}$ lattice, and the level matching condition $\delta^{2}=\frac{1}{2} \bmod \mathbb{Z}$. If one wishes to go to $D=6$, one can decompactify the last $T^{2}$ to get an $N=1$ theory. The six-dimensional anomaly cancellation provides a further test on the analysis of the vacuum given below.

Let us now turn on a field strength on one of the $\mathbb{Z}_{2}$-twisted tori (say $x_{4}$ and $x_{5}$ directions of radii $R_{4}$ and $R_{5}$ ). Decomposing $E_{8}$ in terms of $E_{7} \times S U(2)$, let us take the field strength to be along the Cartan direction of the $S U(2)$. Depending on the choice of the orbifold shift $\delta$, this $S U(2)$ may or may not be broken. In the case when $S U(2)$ is not broken, we have to go to lower dimensions and go to the Coulomb phase where $S U(2)$ is broken down to $U(1)$, in order to discuss the quantization condition for the magnetic flux. Let

us choose a fundamental domain for $T^{2} / \mathbb{Z}_{2}$ as $0 \leq x_{4} \leq R_{4} / 2$ and $-R_{5} / 4 \leq x_{5} \leq 3 R_{5} / 4$ with the identifications on the boundary given by the orbifold group action: the boundary $x_{5}=-R_{5} / 4$ is identified with $x_{5}=3 R_{5} / 4$ and, at the boundaries $x_{4}=0$ and $x_{4}=R_{4} / 2$, $x_{5}$ is identified with $-x_{5}$ for $\left|x_{5}\right| \leq R_{5} / 4$ and with $R_{5}-x_{5}$ for $\left|x_{5}\right| \geq R_{5} / 2$ (see Fig. 1). 

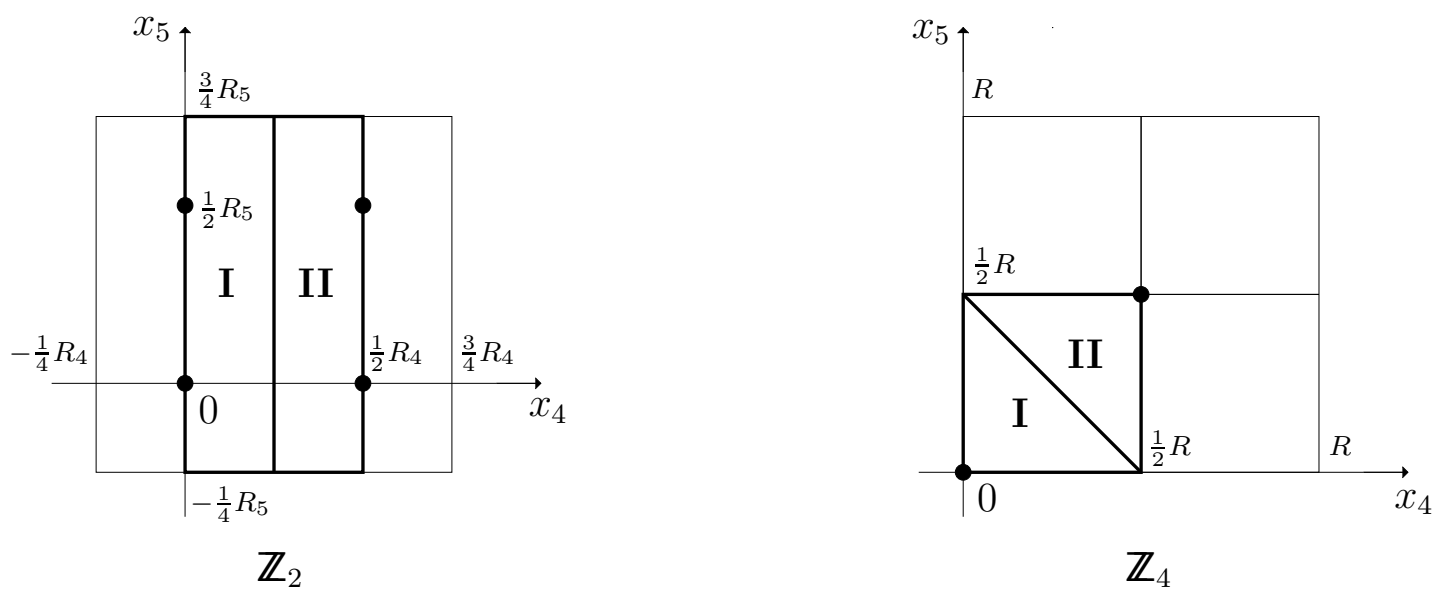

Figure 1: The fundamental domains, $T^{2} / \mathbb{Z}_{2}$ and $T^{2} / \mathbb{Z}_{4}$, for $\mathbb{Z}_{2}$ and $\mathbb{Z}_{4}$ orbifolds, respectively. The two patches, I and II, are defined in the text and the black dots are the fixed points.

The fixed points of the orbifold are at $\left(x_{4}, x_{5}\right)$ given by $(0,0),\left(0, R_{5} / 2\right),\left(R_{4} / 2,0\right)$ and $\left(R_{4} / 2, R_{5} / 2\right)$.

In order to define the gauge potential associated with the $U(1)$ magnetic flux, we choose two patches in the fundamental domain: patch I is given by $0 \leq x_{4} \leq R_{4} / 4$ and patch II by $R_{4} / 4 \leq x_{4} \leq R_{4} / 2$ (see Fig. 1 ). We can now choose a gauge such that the $U(1)$ gauge potential $A_{4}=0$ and $A_{5}=B x_{4} Q$ in patch I and $A_{5}=B\left(x_{4}-R_{4} / 2\right) Q$ in patch II, where $Q$ is the $U(1)$ generator. This choice ensures that, at the boundaries, the gauge potential is well defined under the orbifold group identification mentioned above. The gauge transition function $g_{I I, I}$ in the overlap between the two patches, i.e. at $x_{4}=R_{4} / 4$, is $g_{I I, I}=\exp \left(-i B Q R_{4} x_{5} / 2\right)$. Demanding single valuedness of this transition function under $x_{5} \rightarrow x_{5}+R_{5}$ one obtains the quantization condition $B=\frac{4 \pi n}{q_{\min } R_{4} R_{5}}$, where $q_{\min }$ is the minimum charge and $n$ is an integer. Note that this quantization condition is twice that on the torus and is due to the fact that the area of the orbifold $T^{2} / \mathbb{Z}_{2}$ is half that of the torus $T^{2}$. Taking into account that in our case this $U(1)$ is embedded in an $S U(2)$ and that the charge spectrum also includes the fundamental representation of $S U(2)$ we have that 
$Q=\sigma_{3}$ and $q_{\min }=1$. Thus

$$
g_{I I, I}=\exp \left(-2 i \pi n \frac{x_{5}}{R_{5}} \sigma_{3}\right)
$$

Let us now go to the $S U(2)$ point by suitably adjusting the scalars of the vector multiplets. We would like to show that one can perform $S U(2)$ gauge transformations that respect the orbifold group identifications, such that the transition function $g_{I I, I}$ becomes constant. In general if one performs a gauge transformation by $g_{I}$ in patch I and by $g_{I I}$ in patch II, then the new transition function becomes $g_{I I, I}^{\prime}=g_{I I} g_{I I, I} g_{I}^{-1}$. Let us make the ansatz $g_{I I}=1$ and

$$
g_{I}=h\left(x_{4}\right) e^{-i \pi n \frac{x_{5}}{R_{5}} \sigma_{3}} e^{i f\left(x_{4}\right) \sigma_{1}} e^{i \pi n \frac{x_{5}}{R_{5}} \sigma_{3}} e^{-i f\left(x_{4}\right) \sigma_{1}}
$$

where $f$ and $h$ are smooth and $f(0)=0, f\left(R_{4} / 4\right)=\pi / 2$ and the $S U(2)$ group element $h$ satisfies the condition $\Omega h(0)=h(0) \Omega$, where $\Omega$ is the orbifold group action corresponding to the shift $\delta$. These conditions ensure that $g_{I}$ is well defined on the orbifold. The new transition function then becomes

$$
g_{I I, I}^{\prime}=h^{-1}\left(R_{4} / 4\right) \equiv h_{0}^{-1}
$$

Since $g_{I I, I}^{\prime}$ is a constant, it follows that one can smoothly change the background gauge field strength to zero and as a result one again obtains a supersymmetric vacuum.

We would like to make a couple of remarks here. First, for a gauge transformation $g_{I}$ to exist on patch I such that the new transition function $g_{I I, I}^{\prime}$ is a constant, the quantization condition (3.12), which ensures that $g_{I I, I}$ maps the boundary of patch I into a closed contour on the $S U(2)$ group manifold, is crucial. If, for instance, one considers a theory with no fundamental representations of $S U(2)$, then the quantization condition would imply that $n$ in (3.12) could also be a half-integer. For $n$ half-integer, $g_{I I, I}$ maps the boundary of patch I into an open path in the $S U(2)$ group manifold whose end-points are related by the action of the non-trivial element of the centre $\mathbb{Z}_{2}$. Since the patches are topologically 
discs (due to orbifold identifications), it would be impossible to find a smooth $g_{I}$ such that the new transition function becomes a constant. Consequently the field strength would have been non-zero, giving rise to a cosmological constant. It is quite curious that in string theory, modular invariance guarantees that the spectrum contains also fundamental representations. In turn, this enforces $n$ to be an integer and, as a result, the existence of a new supersymmetric minimum is guaranteed. For the type II theories that are dual to the heterotic string vacua, one can use the perturbative modular invariance on the heterotic side to deduce the charge spectrum of the non-perturbative states in the type II side. It will be interesting to find out whether there is some generalized notion of nonperturbative modular invariance that would impose conditions on the charge spectrum for non-perturbative states for a generic type II theory (that may not have a heterotic dual). This is clearly important, as the existence of the new supersymmetric minimum depends on the quantization condition, and hence on the charge spectrum.

The second remark concerns the role of the tachyon in the above solution (3.14). In order to arrive at the constant transition function, it is necessary that the gauge transformation $g_{I}$ involves also the off-diagonal elements of $S U(2)$. This is so because, for non-zero $n, g_{I I, I}$ maps the boundary of patch I into a closed path in the $U(1)$ subgroup (generated by $\sigma_{3}$ ) that has winding number $n$. This path is non-trivial if one restricts oneself to this $U(1)$ subgroup and becomes trivial only in the $S U(2)$ group manifold; hence $g_{I}$ must necessarily involve off-diagonal elements. Transforming now the original $U(1)$ gauge potential by $g_{I}$ will necessarily introduce an off-diagonal part in the gauge potential; because of the orbifold group invariance of $g_{I}$, this corresponds to turning on expectation values for the tachyon fields (and all the higher Landau levels). The final solution $A=0$ then comes about by a complicated field redefinition using the orbifold group invariant part of the local $S U(2)$ symmetry in six-dimensional space-time.

Just as in the $N=4$ case, the role of the constant group element $h_{0}$ is to turn on 
Wilson lines. To see this, let us extend the fundamental domain of the orbifold to that of the torus, i.e. $-R_{4} / 4 \leq x_{4} \leq 3 R_{4} / 4$. The gauge functions can then be extended by the action of the orbifold group. By combining the transition functions in the two copies of the orbifold fundamental domains, one finds that $x_{4} \rightarrow x_{4}+R_{4}$ is accompanied by the gauge transformation $h_{0}^{-1} \Omega h_{0} \Omega$. If $\Omega$ acts non-trivially on the $S U(2)$ (i.e. $\Omega$ is not in the centre of $S U(2))$ then this is equivalent to turning on an $S U(2)$ Wilson line along the $x_{4}$ direction. Such a Wilson line has the effect of introducing different holonomies around different fixed points [18. In the present example, this amounts to having holonomies $\Omega$ around the fixed points $(0,0)$ and $\left(0, R_{2} / 2\right)$ and $h_{0}^{-1} \Omega h_{0}$ around the other two fixed points. The fact that the gauge transition function $h_{0}^{-1}$ introduces these different holonomies can also be seen by considering the eigenvalue problem for the Laplacian in the $x_{4}, x_{5}$ directions.

Since we have realized the orbifold group action as a $\mathbb{Z}_{2}$ shift, the only non-trivial such $\Omega$ is necessarily of the form $\Omega=i \sigma_{3} \Omega^{\prime}$, where $\Omega^{\prime}$ acts on $E_{7}$ and corresponds to a shift by half the $\mathbf{5 6}$-weight. Note that for this choice of $\Omega$ the roots of $S U(2)$ give rise to hypermultiplets and, as a result, there is no $S U(2)$ enhancement in the four-dimensional theory; instead, what appears at this point is massless hypermultiplets. If $h_{0}$ is not in the $U(1)$ generated by $\sigma_{3}$, then there is a non-trivial Wilson line that can be parametrized by a suitable choice of basis as $e^{i \theta \sigma_{1}}$, where $\theta$ is a continuous parameter. For $\theta=0 \bmod 2 \pi$ there is no Wilson line and the resulting theory is the same as the original one before turning on the flux. Non-zero values of $\theta$ correspond to higgsing the original theory by giving non-zero expectation values to hypermultiplets corresponding to the $S U(2)$ roots.

In fact, for this example, one can construct an explicit CFT description of these models. One can choose a basis where the Wilson line is in the Cartan direction ( say $\left.e^{i \theta \sigma_{3}}\right)$. In this basis the orbifold action $\Omega=i \sigma_{1}$ reflects the Cartan direction. In other words, one obtains an asymmetric orbifold [19], where the $\mathbb{Z}_{2}$ action is defined by reflection of $T^{4}$ together with reflection of the chiral boson $\phi$ corresponding to the Cartan direction of $S U(2)$, and 
a shift in the $E_{7}$ given by half the $\mathbf{5 6}$-weight. Clearly this $\mathbb{Z}_{2}$ is an automorphism of the lattice deformed by the Wilson line: since the latter is along $x_{4}$ in the Cartan direction of $S U(2)$, the deformation of the lattice involves the $x_{4}$ and $\phi$ directions and, as a result, reflecting $x_{4}$ and $\phi$ simultaneously leaves the lattice unchanged. Moreover, it is easy to see that this model satisfies the level matching condition: the original shift of half the fundamental weight of $S U(2)$, as well as the reflection of $\phi$, contribute 1/16 to the level matching condition. In this CFT description, the parameter $\theta$ corresponds to higgsing by giving a vacuum expectation value to the Wilson line that, as a result of orbifold group projection, is part of a hypermultiplet. Thus the new supersymmetric vacuum is in the same class as that of the original vacuum before turning on the magnetic flux. In fact this is not surprising: in the original supersymmetric vacuum, the special points in the vector moduli space, where the untwisted charged states become massless, the latter come in pairs. As a result there is always a flat direction in the Higgs branch. Now after turning on a field strength, if there is a new supersymmetric minimum at non-zero expectation value of the charged states, then clearly the new vacuum is in the original Higgs branch.

Since in the four-dimensional theory the flat $T^{2}$ was a spectator in this entire analysis (apart from providing the Coulomb phase), one can decompactify this to a six-dimensional theory. In this case the original orbifold model gives an $E_{7} \times S U(2)$ gauge group with charged hypermultiplets from the untwisted sector in the $(56,2)$ representation. In the new supersymmetric vacuum, the spectrum can be analysed for different values of the parameter $\theta$. For $\theta=0$ one has the original spectrum, while for $\theta=\pi$ the gauge group is broken to $E_{6} \times U(1)^{2}$ and for generic $\theta$ the gauge group is $E_{6} \times U(1)$, which has lower rank. One can also study the spectrum of massless hypermultiplets and, as expected, the theory is anomaly-free for all values of $\theta$. In fact the point $\theta=\pi$ could have been described in the original basis as an orbifold in the presence of Wilson lines, as studied in Ref. [18]. 


\section{$\mathbb{Z}_{4}$ orbifold}

In the $\mathbb{Z}_{2}$ example considered above, at the special point in the moduli space where extra massless charged hypermultiplets appeared, they did so in pairs, and as a result provided a flat direction. The new vacuum then turned out to be in the same class as the old vacuum before turning on the magnetic field. It is instructive to consider another orbifold example where only one charged massless hypermultiplet appears. In this case there is no flat direction that would break the $U(1)$ in question. In the presence of the magnetic field, if there is a new supersymmetric vacuum, it should be either the same as the original one or a different one, disconnected from the original. This example would therefore be closer in spirit to the one considered in Ref. [四] on the type II side.

Such examples are provided by $\mathbb{Z}_{3}$ or $\mathbb{Z}_{4}$ orbifolds. For notational simplicity we consider here a $\mathbb{Z}_{4}$ orbifold, which is realized by simultaneous $\pi / 2$ rotation in the planes $\left(x_{4}, x_{5}\right)$ and $\left(x_{6}, x_{7}\right)$, together with a shift given by one fourth the weight of $(\mathbf{2 , 5 6 )}$ in the decomposition of $E_{8}$ in terms of $S U(2) \times E_{7}$. For this orbifold, the gauge group is broken to $U(1) \times E_{7}$, with several hypermultiplets transforming under different representations of the gauge group. The hypermultiplets that we are interested in are the ones that are neutral under $E_{7}$ and charged under $U(1)$. In the untwisted sector, this is provided by the $S U(2)$ root and, contrary to the $\mathbb{Z}_{2}$ case, there is just one such hypermultiplet, which we denote by $\Phi$. We can further go to the Coulomb phase by turning on Wilson lines along the $\left(x_{8}, x_{9}\right)$ directions so that $E_{7}$ is broken to $U(1)^{7}$ and all the charged hypermultiplets, including $\Phi$, become massive.

Let us now turn on a magnetic field in the $\left(x_{4}, x_{5}\right)$ plane along the first $U(1)$. The quantization condition can be found, as before, by going to the fundamental domain defined by $0 \leq x_{4} \leq R / 2$ and $0 \leq x_{5} \leq R / 2$ (the two radii are equal as required by $\mathbb{Z}_{4}$ symmetry), see Fig. 1. The two fixed points in this domain are $(0,0)$ and $(R / 2, R / 2)$. We can choose two patches as follows: patch $\mathrm{I}$ is the lower triangle with vertices $(0,0),(0, R / 2)$ and 
$(R / 2,0)$ and patch II is the complementary triangle. Each of these patches is topologically a disc, owing to the $\mathbb{Z}_{4}$ identification of the boundaries: in patch I, the boundary $(x, 0)$ for $0 \leq x \leq R / 2$ is identified with the boundary $(0, x)$; in patch II, the boundary $(R / 2, x)$ is identified with the one defined by $(x, R / 2)$. In other words, various fields on these patches are identified on these boundaries up to a gauge transformation $\Omega$ corresponding to the $\mathbb{Z}_{4}$ shift in the gauge sector defined above (we are considering only fields that do not depend on $x_{6}$ and $x_{7}$, otherwise $\Omega$ will be accompanied by the orbifold group action on these variables). The $U(1)$ gauge potential that gives rise to a constant magnetic field can be defined on the two patches, such that it satisfies the boundary identifications, as

$$
A_{4}^{I}=B x_{5} \sigma_{3} \quad A_{5}^{I}=-B x_{4} \sigma_{3}
$$

in patch I and

$$
A_{4}^{I I}=B\left(x_{5}-\frac{R}{2}\right) \sigma_{3} \quad A_{5}^{I I}=-B\left(x_{4}-\frac{R}{2}\right) \sigma_{3}
$$

in patch II. In the overlap between the two patches, the gauge transition function is $g_{I I, I}=$ $\exp \left[i B R\left(x_{4}-x_{5}\right) \sigma_{3} / 2\right]$. The identification of the points $(0, R / 2)$ with $(R / 2,0)$, and the fact that the spectrum includes the fundamental representation of $S U(2)$, then gives the quantization condition $B=4 \pi n / R^{2}$ for some integer $n$.

At the special points in the vector moduli space where the hypermultiplet $\Phi$ defined above corresponding to $S U(2)$ roots becomes massless, we can perform a gauge transformation $g_{I}$ in, say, patch I with $g_{I}=h g_{I I, I}$ at the overlap of the two patches, and at the boundaries $g_{I}(0, x)=g_{I}(x, 0)=h g_{I I, I}(0, R / 2)$, where $h$ is a constant $S U(2)$ group element. It is clear that such a smooth $g_{I}$ exists owing to the quantization condition of $B$. The identification of the boundaries $g_{I}(0, x)=\Omega g_{I}(x, 0) \Omega^{-1}$ implies that $h$ commutes with $\Omega$ and as a result $h$ is in the $U(1)$ subgroup generated by $\sigma_{3}$. The new transition function $g_{I I, I}^{\prime}=g_{I I, I} g_{I}^{-1}=h$ is constant and therefore admits a zero gauge potential in the two patches. Thus we see that once again at the special point where the charged hypermulti- 
plet $\Phi$ becomes massless (and hence tachyonic in the presence of a magnetic field) one can smoothly take the field strength to zero, thereby obtaining a new supersymmetric vacuum.

In order to understand the nature of the new vacuum, we must consider the holonomies around the fixed points. Just as in the $\mathbb{Z}_{2}$ case, either by extending the fundamental domain to the original torus or by analysing the eigenvalue problem of the Laplacian on the fundamental domain, we can see that if the holonomy around the fixed point $(0,0)$ is $\Omega$, then around the fixed point $(R / 2, R / 2)$ it is $h^{-1} \Omega h$, which is equal to $\Omega$ since $h$ commutes with $\Omega$. Therefore the new vacuum corresponds to having no Wilson lines in the $\left(x_{4}, x_{5}\right)$ plane. Thus we conclude that the new vacuum is in fact the original supersymmetric vacuum before turning on the magnetic field.

Although all of the above discussion was carried out for the $N=2$ theory, it can be extended to $N=1$ orbifold models. For example, one can consider a $\mathbb{Z}_{2} \times \mathbb{Z}_{2}$ orbifold, with the first $\mathbb{Z}_{2}$ as defined above and the second $g$ acting on $x_{6}, x_{7}, x_{8}$ and $x_{9}$ together with a $\mathbb{Z}_{2}$ shift along $x_{4}, x_{5}$ directions satisfying the level-matching condition. The tachyon now appears in the $N=1$ chiral multiplet, which is accompanied by higher Landau levels. It is clear that the analysis given for the $N=2$ case remains unchanged and the new supersymmetric vacuum can again be given a CFT description as a $\mathbb{Z}_{2} \times \mathbb{Z}_{2}$ orbifold. This shows that this mechanism of supersymmetry restoration, at least in some cases, also works for $N=1$ theories. It will be interesting to study this phenomenon in more general situations as well as in the type II duals.

\section{Tachyons without Landau Levels}

Let us now consider the tachyons coming from the twisted sector in heterotic $N=2$ orbifolds, which, as we have noticed in Section 2, do not give rise to towers of Landau levels with masses of order $g_{H}^{2} / V$. Therefore the analysis of the potential to the leading order in $1 / V$ can be carried out by restricting to the quartic terms in the tachyons. Moreover 
the quartic terms that enter in this analysis are zeroth order in $1 / V$ and therefore can be calculated in the supersymmetric theory by setting the magnetic flux to zero. Assuming that the tachyons are charged only under the $U(1)$ gauge generator along which we have the magnetic flux, these quartic terms are given by the $N=2$ D-terms:

$$
\frac{1}{2} g_{H}^{2}\left\{\left[\sum_{a} q_{a}\left(\left|\chi_{a}\right|^{2}-\left|\eta_{a}\right|^{2}\right)\right]^{2}+4\left|\sum_{a} q_{a} \chi_{a} \bar{\eta}_{a}\right|^{2}\right\}
$$

where the index $a$ runs over the different tachyons and $q_{a}$ are the absolute values of their charges.

In Section 2, we discussed the mass terms for tachyons in type I theory arising from 95 strings. In the type I string frame they are given by Eq. (2.3). In order to obtain the corresponding mass formula for the heterotic theory, we have to use the duality relations 20]:

$$
V_{I}=\frac{V_{H}}{g_{H}} \omega_{H}^{-1 / 2} \quad ; \quad g_{I}^{2}=g_{H} \omega_{H}^{-1 / 2}
$$

where $V_{I}$ and $V_{H}$ are the volumes of the tori on which the magnetic flux is turned on, in type I and heterotic theories, respectively; $\omega_{H}$ is the total volume of the six-dimensional internal space in the heterotic theory; $g_{I}$ and $g_{H}$ are the four-dimensional respective string coupling constants. Starting from the mass formula (2.3) and going to the Einstein frame, while using the quantization condition for the magnetic field $F=2 \pi / V_{I}$ in units where we normalize the minimum charge to 1 , we obtain the tachyon mass squared $M^{2}=-|q| g_{I}^{2} / V_{I}$. This is mapped in the heterotic theory, by means of the duality relations (4.2), to $M^{2}=-|q| g_{H}^{2} / V_{H}$. Combining this mass term with the quartic potential (4.1) and the cosmological constant obtained previously in Eq. (3.11) by integrating $F^{2}$ over the internal space, we obtain:

$$
\mathcal{V}_{\text {eff }}=\frac{1}{g_{H}^{2}}\left(\frac{2 \pi}{V_{H}}-\sum_{a} q_{a}\left|\chi_{a}\right|^{2}\right)^{2},
$$

where we have set to zero all the non-tachyonic components $\eta_{a}$ 's.

The above potential has a minimum at $\sum_{a} q_{a}\left|\chi_{a}\right|^{2}=\left(2 \pi / V_{H}\right)^{2}$, at which the cosmological constant vanishes and supersymmetry is restored. Unlike in the previous case, the $U(1)$ 
is spontaneously broken at the new minimum, with its gauge field absorbing one linear combination of hypermultiplets. This is analogous to the type II situation analysed in Ref. [1].

\section{Conclusions}

We would now like to see what the results on the heterotic side imply for the type IIA side. In particular, the first case involving Landau levels studied here should have a counterpart on the type IIA side. In fact, it is not hard to see what these states are since they have a six-dimensional interpretation. They are the 2-branes wrapped around 2-cycles in the $K_{3}$ fibre (which survive the fibration) and carry Kaluza-Klein momenta along the base. Thus these are the original 2-branes boosted along the directions of the base. In the $N=4$ context, these are again BPS states that carry, besides the charge associated to the 2-cycle in $K_{3}$, also the charges corresponding to the Kaluza-Klein $U(1)$ 's coming from the base $T^{2}$. In fact, these BPS states are needed for the heterotic-type IIA duality in order to fill the $O(6,22)$ lattice, and their masses squared due to the Kaluza-Klein momenta (in the Einstein frame) become of order $A+g_{I I}^{2} / V_{I I}$, where $A$ is the area of the 2 -cycle. The limit that we are considering corresponds to $A \rightarrow 0$. In the $N=2$ case, however, since there are no such Kaluza-Klein $U(1)$ 's as the base is $S^{2}$, they are non-BPS states. The masses squared of these states, as in the $N=4$ case, are shifted by an amount of the order of $g_{I I}^{2} / V_{I I}$. In the context of Calabi-Yau spaces, these non-BPS 2-branes should appear through non-holomorphic 2-cycles [21]. Of course, these states are in general unstable; however, they do contribute to the higher-dimensional terms in the effective potential for the BPS states.

This is exactly what happened in the discussion in Section 3 for the heterotic case.

There the quartic coupling was of order $g_{H}^{2}$, while the masses squared were of order $g_{H}^{2} / V_{H}$. As a result, for example, one gets a reducible contribution at the 6-point level (with one 
KK exchange) that behaves as $g_{H}^{2} V_{H}$. On the type IIA side the quartic coupling is $1 / V_{I I}$, and a similar reducible contribution at the $(2 n+4)$-point level due to these non-BPS states is (in the $A \rightarrow 0$ limit) of order $1 /\left(V_{I I} g_{I I}^{2 n}\right)$. This peculiar problem appears because we are taking the limit where $A$ is much smaller than the coupling constant $g_{I I}^{2}$ and, as a result, there is a tower of very light non-BPS states. Thus, in the problem of minimization of the potential in the presence of a magnetic field, these states must also be taken into account. As in the heterotic case, this problem is more easily analysed by going to $D=6$.

For example, let us take the (11,11)-model [5], which is obtained as a $\mathbb{Z}_{2}$ orbifold of $K_{3} \times$ $T^{2}$, with the magnetic field turned on the $T^{2}$. In this model there are two types of special points: one class with an $S U(2)$ enhancement along with an adjoint hypermultiplet; and a second class with an $S U(2)$ enhancement along with four fundamental hypermultiplets. On the heterotic side the first case appears in the untwisted sector, while in the second case the fundamental hypermultiplets arise in the twisted sector. In particular, this means that the charged massless states that appear in the first case have a six-dimensional interpretation. In fact on the type IIA side these states correspond to 2-brane wrapped around one of the vanishing 2-cycles of $K_{3}$, which is even under the $\mathbb{Z}_{2}$ Enriques involution. From the six-dimensional point of view they appear as point particles. Upon further compactification to $D=4$, these particles will in general carry Kaluza-Klein momenta and provide higher Landau levels in the presence of a magnetic field. From the ten-dimensional point of view, they are 2-branes wrapped around vanishing 2-cycles of $K_{3}$ that are boosted along the base. In this case, we can analyse this problem exactly as in Section 3, by going to the six-dimensional theory on $R^{4} \times T^{2} / \mathbb{Z}_{2}$, and we find that the new supersymmetric minimum corresponds to the old vacuum up to non-trivial field redefinitions. In the second case, if we use the tachyons in the fundamental representation, which do not have higher Landau levels in the heterotic theory, then the analysis of Section 4 shows that, in the new supersymmetric vacuum, $S U(2)$ is completely broken by absorbing three hypermultiplets. Thus, in the new vacuum relative to the generic points in the old one, the number of vectors decreases by 1 
while the number of hypermultiplets increases by 5 .

In the $N=2$ case, there are also charged states that have no six-dimensional interpretation in the large volume limit of the base. It is simpler to study these states on the heterotic side compactified on $K_{3} \times T^{2}$. In the orbifold limit, these states come from the twisted sector and do not produce tachyons with Landau levels since they do not carry $K_{3}$ momentum. However, on a smooth $K_{3}$ manifold obtained by switching on the blowing-up modes $\mathcal{B}$ one expects them to have $K_{3}$ Kaluza-Klein excitations in the limit of volume of the base $V_{H} \gg \mathcal{B}^{-2}$. By duality the same behaviour would therefore be expected for type II on Calabi-Yau, which is a $K_{3}$ fibration. Hence, apart from special points such as orbifolds, we expect that in a generic compactification there are Landau levels. A correct treatment should therefore also include these states.

\section{Acknowledgements}

We would like to thank M. H. Sarmadi and G. Thompson for useful discussions. T.R.T. acknowledges the hospitality of the Ecole Polytechnique at the initial stage of this work and of the CERN Theory Division during its completion. 


\section{References}

[1] J. Polchinski and A. Strominger, Phys. Lett. B 388 (1996) 736.

[2] C. M. Hull and P. K. Townsend, Nucl. Phys. B 438 (1995) 109.

[3] E. Witten, Nucl. Phys. B 443 (1995) 85.

[4] S. Kachru and C. Vafa, Nucl. Phys. B 450 (1995) 65.

[5] S. Ferrara, J. Harvey, A. Strominger and C. Vafa, Phys. Lett. B 361 (1995) 59.

[6] A. Klemm, W. Lerche and P. Mayr, Phys. Lett. B 357 (1995) 313.

[7] H. B. Nielsen and P. Olesen, Nucl. Phys. B 144 (1978) 376.

[8] C. Bachas, A way to break supersymmetry, hep-th/9503030.

[9] E. Kiritsis and C. Kounnas, Nucl. Phys. B 456 (1995) 699.

[10] A. Aboulsaood, C.G. Callan, C.R. Nappi and S.A. Yost, Nucl. Phys. B 280 [FS18] (1987) 599 .

[11] M. Bianchi and A. Sagnotti, Phys. Lett. B 247 (1990) 517; Nucl. Phys. B 361 (1991) 519.

[12] E. Gimon and J. Polchinski, Phys. Rev. D 54 (1996) 1667.

[13] A. Dhabolkar and J. Park, Nucl. Phys. B 477 (1996) 701.

[14] E. Gava, K. S. Narain and M. H. Sarmadi, On the bound states of $p$ - and (p+2)-branes, hep-th/9704006.

[15] J. Ambjorn and H. Flyvbjerg, Phys. Lett. B 97 (1980) 241.

[16] G. 't Hooft, Commun. Math. Phys. 81 (1981) 267. 
[17] M. A. Walton, Phys. Rev. D 37 (1988) 377.

[18] L. E. Ibañez, H. P. Nilles and F. Quevedo, Phys. Lett. B 187 (1987) 25.

[19] K. S. Narain, M. H. Sarmadi and C. Vafa, Nucl. Phys. B 288 (1987) 551 and Nucl. Phys. B 356 (1991) 163.

[20] I. Antoniadis, C. Bachas, C. Fabre, H. Partouche and T. R. Taylor, Nucl. Phys. B 489 (1997) 160.

[21] K. Becker, M. Becker and A. Strominger, Nucl. Phys. B 456 (1995) 130. 